\title{
Article \\ Knowledge Development Trajectories of the Radio Frequency Identification Domain: An Academic Study Based on Citation and Main Paths Analysis
}

\author{
Wei-Hao Su ${ }^{1, *}$, Kai-Ying Chen ${ }^{1}$, Louis Y. Y. Lu ${ }^{2}$ and Jen-Jen Wang ${ }^{1}$ \\ 1 Department of Industrial Engineering and Management, National Taipei University of Technology, 1, Sec. 3, \\ Zhongxiao E. Rd., Taipei 10608, Taiwan; kychen@mail.ntut.edu.tw (K.-Y.C.); wangju1129@gmail.com (J.-J.W.) \\ 2 College of Management, Yuan Ze University, 135 Yuan-Tung Road, Chung-Li 32003, Taiwan; \\ louislu@saturn.yzu.edu.tw \\ * Correspondence: jackiesu1969@yahoo.com.tw; Tel.: +886-975527516
}

Citation: Su, W.-H.; Chen, K.-Y.; Lu, L.Y.Y.; Wang, J.-J. Knowledge Development Trajectories of the Radio Frequency Identification Domain: An Academic Study Based on Citation and Main Paths Analysis. Appl. Sci. 2021, 11, 8254. https:// doi.org/10.3390/app11188254

Academic Editor: Federico Divina

Received: 13 August 2021

Accepted: 4 September 2021

Published: 7 September 2021

Publisher's Note: MDPI stays neutral with regard to jurisdictional claims in published maps and institutional affiliations.

Copyright: (c) 2021 by the authors. Licensee MDPI, Basel, Switzerland. This article is an open access article distributed under the terms and conditions of the Creative Commons Attribution (CC BY) license (https:/ / creativecommons.org/licenses/by/ $4.0 /)$.
Featured Application: This study provides planning directions for industry, and the findings serve as a reference for business domain.

\begin{abstract}
The study collected papers on radio frequency identification (RFID) applications from an academic database to explore the topic's development trajectory and predict future development trends. Overall, 3820 papers were collected, and citation networks were established on the basis of the literature references. Main path analysis was performed on the networks to determine the development trajectory of RFID applications. After clustering into groups, the results are twenty clusters, and six clusters with citation counts of more than 200 were obtained. Cluster and word cloud analyses were conducted, and the main research themes were identified: RFID applications in supply chain management, antenna design, collision prevention protocols, privacy and safety, tag sensors, and localization systems. Text mining was performed on the titles and abstracts of the papers to identify frequent keywords and topics of interest to researchers. Finally, statistical analysis of papers published in the previous 4 years revealed RFID applications in construction, aquaculture, and experimentation are less frequently discussed themes. This study provides planning directions for industry, and the findings serve as a reference for business domain. The integrated analysis successfully determined the trajectory of RFID-based technological development and applications as well as forecast the direction of future research.
\end{abstract}

Keywords: radio frequency identification (RFID); citation network analysis; development trajectory; knowledge discovery and extraction; data mining

\section{Introduction}

The term radio frequency identification (RFID) was first proposed in a project led by the Massachusetts Institute of Technology Auto ID Center in 1948. However, academic and industrial research on RFID did not increase in popularity until the 1990s [1]. Since 2000, numerous research papers on RFID have been published. By 2005, RFID-related technologies had matured, with the relevant literature and number of patents having increased considerably. Throughout RFID development, technological advancements and cost reductions have made RFID applications more feasible, including Internet of Things (IoT; also Industry 4.0) products and technologies [2]. A research report on global manufacturers published by the ARC Advisory Group suggested that RFID technology is crucial to increasing IoT-related production capacity [3]. RFID is clearly a key technology in Industry 4.0 and is fundamental to the development of smart factories.

Mature RFID technology provides manufacturers with increased production capacity and lower manufacturing costs. In the 1990s, RFID was applied in manufacturing, including 
production automation, warehouse management, transportation monitoring, and asset management. In recent years, RFID has been incorporated into various smart sensor applications, including internet protocol security, smart maintenance, unstaffed Tencent shops, and self-checkout systems at Japanese convenience stores. Additionally, RFID systems have been combined with database management systems, computer networks, and firewalls to provide automated, safe, and convenient real-time monitoring, such as for security, flight passenger baggage transport, and health care management systems.

Because of its development of IoT technology, China [4] has become the largest market for RFID systems, boasting a market scale of more than US\$6.6 billion. Data analysis conducted by DTechEx [5] to forecast the size of the global RFID market predicted a 7.7\% compound annual growth rate from 2017 to 2023 and that by 2023, the RFID market would be worth US\$31.42 billion (Figure 1). The main reasons for this predicted growth are mergers and acquisitions, alliances, and joint development strategies among enterprises in the RFID ecosystem. In particular, RFID technology is commonly used to connect smart and nonsmart devices as a means of achieving maximum value in the IoT industry. With an increase in demand among emerging economies seeking to industrialize, various RFID technologies have become popular in industry.

\section{RFID MARKET FOR TAGS 2014-2025(\$ BILLION)}

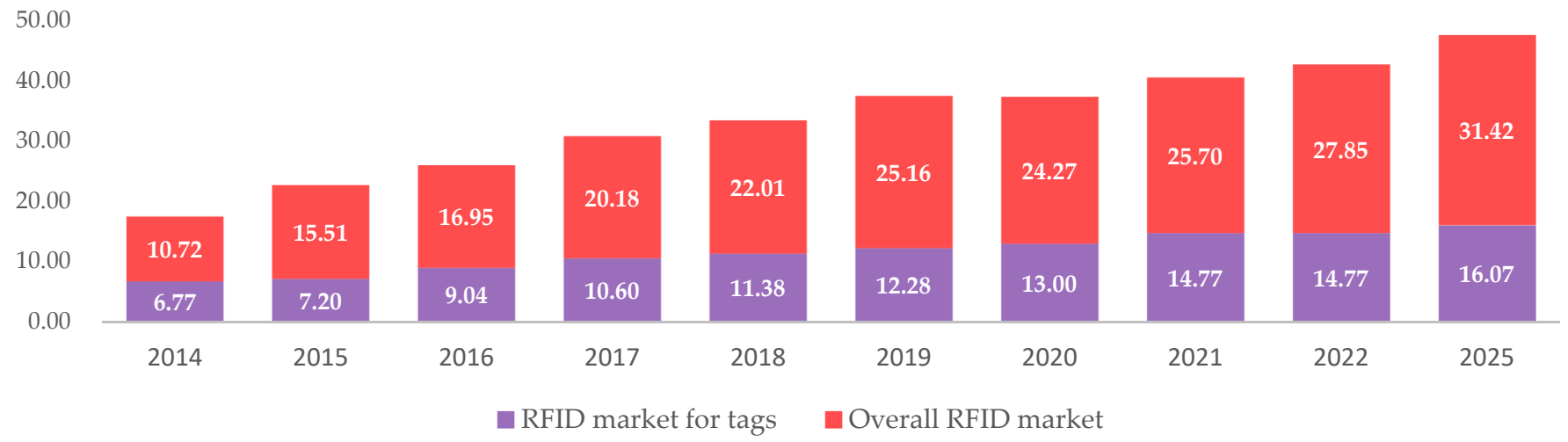

Figure 1. Worldwide Radio Frequency Identification (RFID) revenue [3].

Their literature review focused on the use of RFID technology in specific fields and indicated that RFID is commonly applied in supply chain management and the manufacturing industry. In particular, antennas design for use in RFID localization algorithms have been widely developed for various applications. The application of RFID in other fields, such as collision prevention and RFID-integrated tag sensing in 2014. Due to the limitation of the analysis method used, scholars can only include a few specific literature reviews, and consequences cannot be shown comprehensively. Although these literature reviews can provide research results from the past, there is always the problem of not being able to get the overall trend of development. The main paths uncover the dynamic development process along the timelines, which provide a systematic and effective way for readers to fully grasp the knowledge diffusion trajectories of these domains.

The purpose of this research has the following three points: (i) to identify future development trends of RFID by using the traversal count algorithm in main path analysis; (ii) the results can predict the future technological development through text mining and growth curve analysis; (iii) the results describe major technological patterns and significant commercial value after clustering.

The purpose of this research can be described in three points: (i) to identify future development trends of RFID by using the algorithm in main path analysis; (ii) the results can predict the future technological development through text mining and growth curve analysis; and (iii) the clustering results understand major technological patterns, and utilized in the business environment to do with significant business contribution. 


\subsection{Identifying Core Academic Literature}

RFID was used for aircraft identification during World War II. Walmart, the largest retailer in the United States, required its 100 most important pharmaceutical suppliers to embed RFID in all products by the end of 2006 with the aim of accelerating product identification. This prompted a trend in RFID application in multiple industries. RFID is a contactless, automated identification technology that employs electronic tags, reader devices, and radio waves. The electronic tag, smaller than $2 \mathrm{~mm}$ in diameter, is the key component. A radio wave can be emitted to an electronic tag from a distance of millimeters to meters, and a sensor can then read information embedded in the tag, thus identifying the product, device, or personnel. On the basis of their operating frequency, RFID readers can be classified into low-frequency, high-frequency, ultra-high-frequency (UHF), and microwave readers. Similarly, electronic tags can be divided into passive, semi passive, and active tags depending on the degree to which they are semi battery powered [6].

The papers that form key nodes in a citation network are crucial in their field and are often referenced by researchers. The present authors reviewed the papers in the major paths of the citation network relating to RFID. Karthaus and Fischer [7] proposed a fully integrated passive UHF RFID transponder integrated circuit with 4.5- or 9.5-m reading distance at $500-\mathrm{mW}$ effective radiated power $(16.7-\mu \mathrm{W}$ radio frequency input power) or 4 -W effective isotropic radiated power (EIRP), respectively. The reader was capable of operating in the $868 / 915-\mathrm{MHz}$ ISM band with an antenna gain of less than $0.5 \mathrm{~dB}$. In terms of the received power level required for a given base-station transmission power and tag antenna gain, the proposed reader outperformed other RFID readers by a factor of three. On the basis of the work by Karthaus and Fischer [7], Curty et al. [8] developed a remotely powered UHF RFID transponder operable at $2.45 \mathrm{GHz}$. The system achieved a 12 -m operating range at 4-W EIRP in base-station transmission. When the reader design was fabricated using silicon on sapphire and embedded in a rectifier, the resistance between the tag antennas was reduced. This system represented a crucial improvement on the tag reader proposed by Karthaus and Fischer [7]. Marrocco [9] used the human body as an object to be tagged or biomonitored and designed a geometrical tag antenna on a suspended patch and fed via a nested slot to study the compatibility of antennas with the human body. Building on this work, Marrocco [10] reduced the size of the system and embedded electronic components to reduce the resistance. Additionally, researchers have suggested using three-dimensional spatial algorithms to simulate real environments; in applying the algorithm to automated indoor transportation devices, the deviation between the reader and tag antenna could be optimized [11].

Yang et al. [12] revealed that sparsely distributing RFID tags results in higher localization accuracy than densely distributing them does. However, most researchers have proposed that the RFID tag density be increased or the localization algorithm be modified to improve the positioning accuracy. Zhang et al. [13] employed the simultaneous localization of multiple targets and received signal strength indicators (RSSIs) to test indoor localization using passive UHF RFID based on tag interaction analysis; a revised algorithm that was robust to different numbers, spacing, and materials of target objects was then devised to improve the localization accuracy. Researchers have also applied RFID tags to solve localization problems. Yang [14] experimented with a passive RFID localization system and determined that it had higher localization precision than that of other passive RFID localization systems. Given that RFID uses unique identifiers, enables traceability, and has high security, and Ray et al. [15] proposed the application of RFID to traceability and assurance protocols within the IoT to track and ensure the safety of products. Additionally, the tags are not physically clonable, and the use of fake tags can be prevented by the use of security policy description language.

Gao et al. [16] employed a fingerprint positioning algorithm to receive signals and a nonmetric multidimensional scaling algorithm to obtain the location of a tag. Marindra and Tian [17] proposed the use of weighted multidimensional scaling for multitag cooperative localization and used RSSIs to determine the distance between the reader and 
tags; higher accuracy was achieved when they used weighted multidimensional scaling compared with other approaches. To increase a system's single-target localization accuracy, Rehman et al. [18] proposed an RFID tag array in which laser measurements are employed to localize moving objects and individuals. By using density-based spatial clustering of applications conducted using a noise algorithm, the researchers determined the method to be $25 \%$ more accurate than the conventional method-tag-based localization.

\subsection{Define the Overall Development Direction of Studies on RFID}

The above review literature is only a collection of limited literature on RFID-related technologies in a specific field, although we can know more about this field, but cannot understand the overall development trajectory of RFID. Lan et al. [19] discussed a literature review of RFID-related papers from 1997 to 2011 in the USPTO database to find out the development trajectory of RFID by using main path analysis, but they did not explore the academic literature on Web of Science (WOS). Papers related to RFID technology research were collected from the Web of Science database from 1995 to 2019. Scholars have not yet provided a comprehensive view on the RFID-related literature, compiled a list of RFID technologies that have been studied, or identified the development or market potential of RFID. Determination of the overall trajectory of RFID development could help fill gaps in RFID-related research and identify the development potential and future research directions for RFID. Over recent decades, most studies have adopted similar perspectives to investigate the development of RFID. Therefore, the present study collected numerous academic papers on RFID to analyze academic knowledge diffusion among industries and adopted innovative methods to investigate the future development track of RFID.

In this study, a main path analysis of the critical path (Liu and Lu, 2012), was adopted to define the overall development direction of studies on RFID. For a more rigorous evaluation and analysis of the consistency between corresponding clusters, keywords were used to identify specific development titles. Moreover, technical analysis of text and data was combined with growth analysis. Finally, a key-route main path analysis of the critical path was applied to analyze the connection between the development tracks and the clusters in order to derive a more insightful literature review and analysis.

\section{Materials and Methods}

This study employed main path analysis, clustering, and a word cloud to comprehensively determine the development trajectory of RFID technology. In main path analysis, the citation relationships between papers were explored to examine the overall development trajectory. Next, clustering was used to identify the main research themes. Finally, a word cloud was constructed to determine the frequency at which some terms appeared in paper titles and abstracts. Through these steps, the main RFID-related research issues addressed by scholars were determined.

\subsection{Citation-Based Main Path Analysis}

Main path analysis is a mathematical tool, first proposed by Hummon and Doreian in 1989 , for identifying the major paths in a citation network, which is a type of directed acyclic graph [20]. Hummon and Doreian used the traversal count in main path analysis to describe the citation network of papers related to the development of a specific scientific field.

The goal of this type of analysis is to identify the strongest citation relationship among relevant papers in a scientific field to identify future development trends. Batagelj [21] proposed a new traversal count — the search path count (SPC) — and recommended the SPC over the search path link count and search path node repair because of its "nice" properties. The SPC weight of a selected link is the number of all paths from source to sink that pass through this link. A source is defined as a node that is cited but cites no other nodes, whereas a sink is defined as a node that cites other nodes but is not cited. Search algorithms are used to identify all possible paths between the sources and sinks of citation networks, and the traversal count between each pair of nodes is calculated. The 
path formed by the nodes with the highest traversal count is identified as the main path. In the present analysis, the citation network has sources A and B and sinks $\mathrm{H}, \mathrm{J}$, and I, as illustrated in Figure 2. The black lines represent the main paths, which were determined through the following procedure:

1. The link with the largest traversal count is identified from all possible links emanating from all sources.

2. The link with the largest traversal count emanating from the current start notes is found.

3. Step 2 is repeated until a sink is reached.

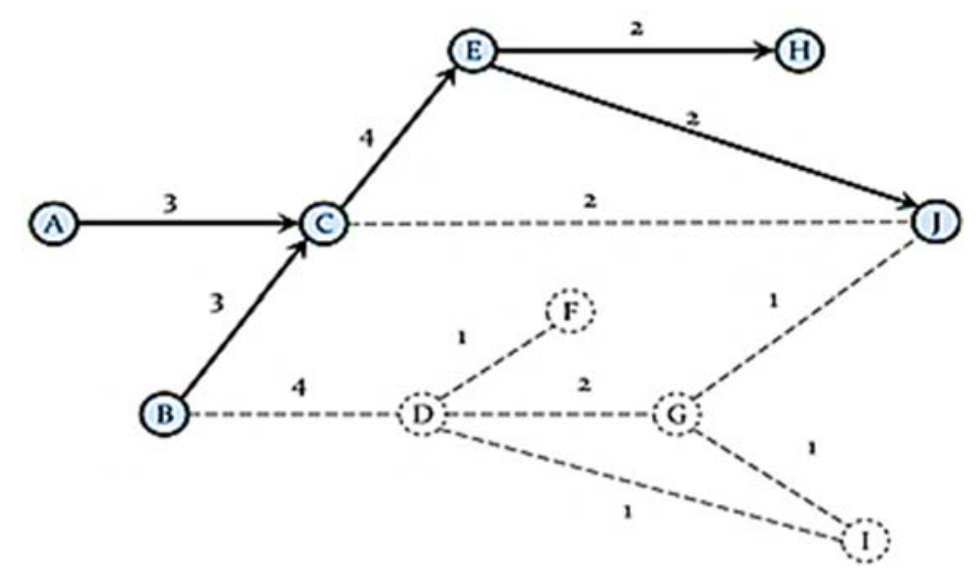

Figure 2. Schematic of the calculation of link weight by using the search path count (SPC).

Liu and Lu [22] employed two models of main paths, namely the global main path and the key-route main path, to elucidate the overall trajectory of knowledge diffusion. Key-route main paths reveal information on the branching of theory development and indicate the process of paradigm shift. Numerous scholars have successfully used main path analysis and key-route main path analysis to review scientific or technical papers [23-27]. Su et al. [28] successfully plotted the trajectory of AR technology development and applications and predicted future patent research and development directions. Other scholars have performed literature reviews of patent data by using main path analysis [29-31].

\section{2. $h$-Index and g-Index}

The $h$-index [32] and $g$-index [33] are author-level metrics that measure both the productivity and citation impact of a scholar's publications and have been proposed to be meaningful mixed quantification indicators. The $h$-index indicates that an author has published $h$ papers that have each been cited at least $h$ times. The $h$-index is calculated on the basis of the distribution of citations of a given researcher's publications, such that given a set of articles in decreasing order of the number of citations they have received, the $g$-index is the unique largest number such that the first $g$ articles received a total of at least $g^{2}$ citations. Because citations from more frequently cited papers are used to bolster the importance of lower-cited papers in the $g$-index, the $g$-index is more effective than the $h$-index in indicating the significance of a paper.

\subsection{Edge-Betweenness Clustering}

Girvan and Newman (2002) proposed an edge-betweenness clustering technique for network grouping. The betweenness of an edge is the number of shortest paths between pairs of vertices that run along the edge. Edge-betweenness can be used to split a citation network into several groups [34]. Figure 3 illustrates the concept of edge-betweenness clustering. When links a, b, c, and d are removed, three groups can clearly be identified. In a network, two nodes can be considered similar, or structurally equivalent, if they have identical links with all the other nodes. In the context of this study, this means that two 
articles have similar topics if they not only cite the same set of articles but are also cited by another identical set of papers. On the basis of this assumption, Newman and Girvan (2004) demonstrated that edge-betweenness clustering is a feasible and useful approach to grouping nodes within a large-scale citation network [35,36]. Newman $(2006)$ further recommended the concept of modularity when determining the optimal structure of a network [37]. Modularity is defined as "the number of edges falling within groups minus the expected number in an equivalent network with edges placed". The optimal division of a network is the one that produces the largest network modularity.

In a network, two nodes can be considered similar, or structurally equivalent, if they have identical links with all the other nodes. Under the context of this study, that means two articles pursue similar topics if they not only cite the same set of literature, but also are cited by another identical set of literature. Cluster analysis is to find several groups with a certain common characteristic from a large amount of original data. In each group, the members of the group have very similar characteristics, but the members of the groups are quite different in this characteristic. In other words, the variation within a group is relatively smaller than the variation between groups.

In the RFID field, many papers discuss all of supply chain management, antenna design, and tag sensors. Moreover, clustering papers according to keywords is difficult. Edge-betweenness clustering is a citation-based approach to dividing a citation network into groups within which the network connections are dense but between which the network connections are sparse.

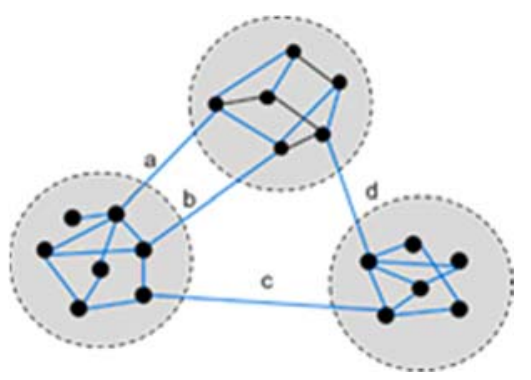

Figure 3. Illustration of edge-betweenness clustering.

\subsection{Word Clouds}

In this study, the frequency with which a keyword appeared in the abstracts and titles of papers was calculated to determine its importance in the RFID field and to identify the main RFID-related research topics discussed in academia during a specific period. A word cloud is a novel visual representation of textual data and a means of visualizing free-form text [38].

\subsection{Growth Curve Analysis}

S-shaped curves are often used to analyze time-series data. These curves illustrate the growth pattern of time-series data. Growth in the body of scientific literature is assumed to exhibit an S-shaped curve; growth stops when the peak of the curve is reached. S-shaped curves comprise four stages, namely the sprouting, growing, maturing, and saturation stages; thus, these curves are also called growth curves. In this study, the growth curve was not used to analyze the market of a product. Instead, this curve was used to analyze the growth of RFID technology and the life cycle of RFID development [39].

\section{Results}

\subsection{Data Collection and Keyword Retrieval}

In this study, we used a set of keywords to retrieve relevant papers from the Web of Science (WOS) database. The keyword set "RFID" and "radio frequency identification" was used to retrieve papers and ensure they were related to RFID technology. The 
research basis; authors; and year, journal, journal volume, and journal page of publication of 4173 RFID-related papers were obtained. After erroneous data-including blank data, garbled text, and papers with anonymous authors-were screened out, 3820 data points remained.

From 1995 to 2019, growth was observed in the yearly number of RFID-related papers published (Figure 4). However, from 2015 to 2016, the yearly number of RFID-related papers published decreased slightly. This was mainly because most papers collected from the WOS database and published during these years contained errors or had no data in the author, title, or publication year column.

Cumulative number of RFID-related papers

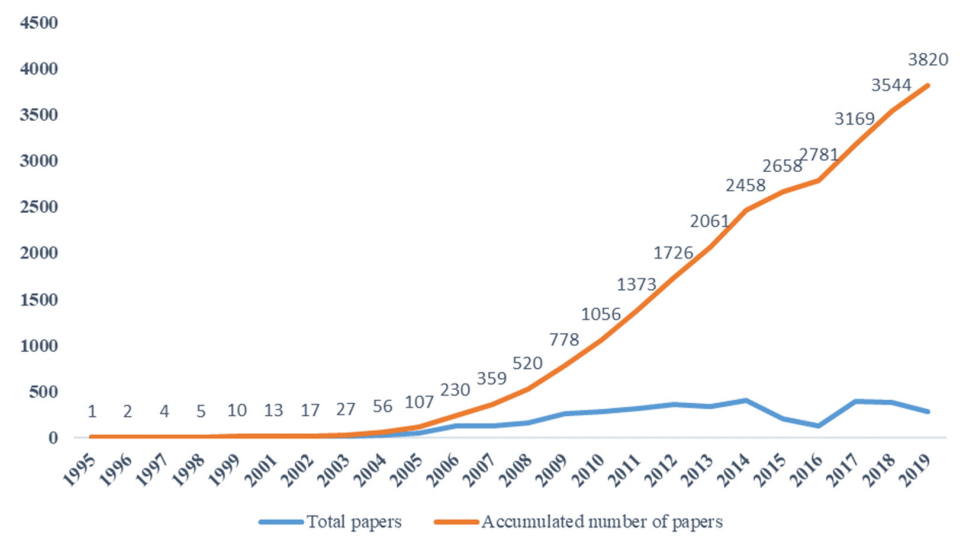

Figure 4. Cumulative number of RFID-related papers.

\subsection{Journal Statistics}

After searching for papers in the WOS database by using keywords, and collecting the MainPath log, we ranked the 20 most influential journals in the RFID field in accordance with their $g$-index. If journals had identical $g$-indexes, the $h$-index was used as a secondary ranking indicator (Table 1). The RFID-related papers were published in 903 academic journals. The most influential journal (according to the g-index), IEEE Transactions on Antennas and Propagation, published 126 papers related to RFID. This journal reports research on and applications of antenna technology and electromagnetic wave transmission. The secondhighest-ranking journal, which was ranked first in terms of its $h$-index, was IEEE Antennas and Wireless Propagation Letters. The theme of this journal, which published 148 RFIDrelated papers, is antennas and wireless transmission fields. The third- to fifth-ranking journals were the International Journal of Production Economics, Automation in Construction, and IEEE Transactions on Industrial Electronics; these journals focus on the economy of manufacturing, the use of automation in construction, and the trading of industrial electronic products, respectively. The other journals among the 20 most influential journals in the RFID field included IEEE Transactions on Instrumentation and Measurement, the Journal of Medical Systems, and Computers and Electronics in Agriculture, which focus on instrumental measurements, medical systems, and agricultural electronics and systems, respectively. Notably, IEEE Sensors Journal and Sensors respectively ranked sixth and tenth in the $g$-index ranking (fourth and ninth in the $h$-index ranking) and published 72 and 100 papers on the topic of sensors. However, neither published articles that were highly cited. In the future, researchers can observe the change in ranking of these two journals to determine whether they have become more important. 
Table 1. Top 20 most influential journal in RFID field.

\begin{tabular}{|c|c|c|c|c|c|c|}
\hline$g$-Index Ranking & Journal & $g$-Index & $h$-Index & Active Years & $\begin{array}{c}\text { Papers } \\
\text { after } 2000\end{array}$ & $\begin{array}{c}\text { Total } \\
\text { Papers }\end{array}$ \\
\hline 1 & $\begin{array}{l}\text { IEEE Transactions on Antennas } \\
\text { and Propagation }\end{array}$ & 52 & 29 & 2003 2019 & 126 & 126 \\
\hline 2 & $\begin{array}{l}\text { IEEE Antennas and Wireless } \\
\text { Propagation Letters }\end{array}$ & 44 & 32 & 2003 2019 & 148 & 148 \\
\hline 3 & $\begin{array}{l}\text { International Journal of } \\
\text { Production Economics }\end{array}$ & 40 & 27 & 2006 2019 & 40 & 40 \\
\hline 4 & Automation in Construction & 40 & 22 & 2007 2019 & 37 & 45 \\
\hline 5 & $\begin{array}{l}\text { IEEE Transactions on } \\
\text { Industrial Electronics }\end{array}$ & 37 & 22 & 2005 2018 & 45 & 37 \\
\hline 6 & IEEE Sensors Journal & 34 & 25 & 2007 2019 & 72 & 72 \\
\hline 7 & Expert Systems with Applications & 33 & 19 & $2006 \sim 2018$ & 42 & 42 \\
\hline 8 & $\begin{array}{l}\text { IEEE Transactions on } \\
\text { Instrumentation and Measurement }\end{array}$ & 33 & 17 & 2006 2019 & 40 & 40 \\
\hline 9 & $\begin{array}{l}\text { IEEE Transactions on Automation } \\
\text { Science and Engineering }\end{array}$ & 31 & 15 & 1999 2019 & 27 & 43 \\
\hline 10 & Sensors & 29 & 17 & 2009 2019 & 100 & 100 \\
\hline 11 & $\begin{array}{l}\text { Computers and Electronics } \\
\text { in Agriculture }\end{array}$ & 28 & 14 & $2004 \sim 2018$ & 28 & 50 \\
\hline 12 & Electronics Letters & 26 & 14 & 1999 2019 & 50 & 29 \\
\hline 13 & Advanced Engineering Informatics & 25 & 15 & 2008 2019 & 21 & 40 \\
\hline 14 & Journal of Medical Systems & 24 & 15 & 2009 2018 & 40 & 27 \\
\hline 15 & $\begin{array}{l}\text { IEEE Transactions on } \\
\text { Industrial Informatics }\end{array}$ & 23 & 16 & 2007 2019 & 23 & 23 \\
\hline 16 & $\begin{array}{l}\text { IEEE Antennas and } \\
\text { Propagation Magazine }\end{array}$ & 22 & 14 & 2008 2018 & 22 & 22 \\
\hline 17 & $\begin{array}{c}\text { IEEE Transactions on Microwave } \\
\text { Theory and Techniques }\end{array}$ & 21 & 15 & 2007 2017 & 42 & 21 \\
\hline 18 & $\begin{array}{l}\text { IEEE/ACM Transactions } \\
\text { on Networking }\end{array}$ & 21 & 14 & 1999 2019 & 26 & 27 \\
\hline 19 & IEEE Journal of Solid-State Circuits & 21 & 13 & $2003 \sim 2018$ & 21 & 21 \\
\hline 20 & $\begin{array}{l}\text { Industrial Management \& Data } \\
\text { Systems information }\end{array}$ & 20 & 13 & 2005 2017 & 20 & 20 \\
\hline Total & & & & & & 973 \\
\hline
\end{tabular}

\subsection{Academic Literature and the Overall Development Trajectory of RFID}

Key-route main path analysis was performed to explore the overall development trajectory of the RFID-related literature and identify the key academic research on RFID. Papers on RFID localization and tracking algorithms were the main path. From initial designs of low-power passive RFID structures to RFID structures with improved reading zones and functions for localizing smaller tags, various algorithms have been proposed. In Figure 5, the green and blue nodes represent the start and link nodes, respectively, and thicker paths indicate a stronger influence. Each node is represented by the last name of the first author, the last initials of the coauthors, and the year of publication. The analysis revealed that 46 papers were frequently cited. The papers were then analyzed in accordance with their publication year in the following sections (Figure 5). 


\subsubsection{Group 1: Supply Chain Management (Period: 1991-2011)}

The path on the lower right side of Figure 5 represents papers on supply chain management. Since the beginning of RFID technology application in the supply chain and manufacturing industry, studies have been conducted on management cost reduction and perceived risk analysis before and after RFID implementation. The papers discuss RFID applications in automation control, library management, food processing, construction components, and paper crafts. Other studies have performed literature reviews and comprehensive literature analyses of papers published in 1991-2005 concerning RFID applications in supply chains. The potential development paths of RFID applications include supply chains, health care, and individual privacy [40]; the incorporation of RFID into logistics [41]; the cost factors influencing the performance of RFID supply chains [42]; the incorporation of RFID into lean production management to save $81 \%$ of operation time [43]; and assessment of the influence of RFID on supply chain performance [44].

Ding et al. [45] developed an RFID-enabled social manufacturing system that enables corporations to perform real-time monitoring of production and transportation missions and assess logistical performance by using collected data. Kang et al. [46] proposed an RFID-based sequencing-error-proofing system to optimize automotive manufacturing, thereby eliminating idle time due to manufacturing errors and component shortages. These studies indicate that RFID can be used to simultaneously and rapidly identify multiple targets and provide information storage advantages that are more favorable than those provided by barcode-based identification.

\subsubsection{Group 2: Antenna Design (Period: 2008-2014)}

The path on the center left is the antenna design path. Antennas have been applied in various fields within the past 16 years, and new RFID localization algorithms have been continually developed. The center path is the global main path of this study, and it has the greatest weight. Because antenna structures and environmental factors determine antenna performance, RFID applications in antenna design are crucial technological improvements. Studies have proposed various means of improving the properties of antennas, such as employing a planar inverted-F antenna with metal coating to prevent reflection [47] and using C-shaped coupling slots for dual-polarized aperture-coupled circular patch antennas [48]. Rao et al. [49] proposed a distance measurement-based antenna design for tracking tags in warehouse storage. Chen and Lin [50] designed a metal tag with a high-impedance surface structure and a small, ultrathin antenna. Yan et al. [51] solved the bending and curving problems with traditional tags by using broadside radiation as the optimal radiation mode for a $45^{\circ}$ main beam direction.

\subsubsection{Group 3: Collision Prevention Protocols (Period: 2010-2015)}

The lower left path in Figure 5 represents research into RFID frequency and applications of RFID-based collision prevention protocols. Papers in this path present research on the ultra-wideband (UWB) RFID tags proposed by Hu et al. [52]. Dardari et al. [53] posited that UWB technology overcomes various limitations of current RFID technology, suggesting that UWB RFID will become the main theme in the RFID field. The study also concluded that protocols can be divided into those preventing tag-reader collision versus those preventing reader-reader collision. These protocols respectively aim to solve localization errors when one reader is used to scan multiple tags and when one tag receives return signals from multiple readers [54]. Studies on the improvement of signal communication have employed the signal-to-interference-plus-noise ratio to reduce the interference range of readers [55], defined the interrogation-range reduction ratio and tag reading range of readers [56], and employed the efficient reader anticollision algorithm of polling servers [57]. Furthermore, enabling readers to rapidly determine whether a system can operate normally and is free of interference greatly reduces the frequency of reader-reader collision. 
One study employed a probabilistically distributed color selection collision prevention algorithm in multiple transmission channels to reduce the occurrence of reader-reader collision by more than $30 \%$. The neighbor friendly reader anticollision (NFRA) protocol is capable of achieving optimal signal communication capacity but does not consider the long duration of signal transmission between tags, resulting in signal delays in the reader. Ferrero et al. [58] proposed the use of balanced throughput to ensure the signal communication capacity of each reader. Gandino et al. [59] discovered that the distributed color noncooperative selection protocol achieved a $16 \%$ greater signal communication capacity than the NFRA protocol did. Golsorkhtabaramiri et al. [60] suggested using RFID tags with independent control signal communication channels to prevent interreader collision and to eliminate repeated data. Golsorkhtabaramiri and Issazadehkojidi [61] created a new collision avoidance protocol with a higher signal communication capacity than that of the NFRA protocol, and it adhered to European standards and regulations. In brief, signal collision reduces signal communication capacity, lowers system performance, and consumes considerable power. Accordingly, Golsorkhtabaramiri et al. [62] evaluated the energy consumption of protocols for reader collision avoidance to determine the most efficient application for preventing reader collision. Liu et al. [63] calculated the offloading capacity of IoT devices to analyze the power consumption of components in mobile RFID systems and proposed a model to reduce system power consumption.

\subsubsection{Group 4: Tag Sensors (Period: 2014-2019)}

The upper left path consists of papers published after 2014 on the new applications of RFID-integrated tag sensors. Digiampaolo and Martinelli [64] employed a distance sensor mounted on RFID readers for the localization of robot positions and the research and development of various sensor applications, including temperature measurements and the detection of metal cracking. RFID-based sensor applications are strongly correlated with RFID applications in chemistry. Scientists have used RFID to detect, identify, and quantify chemical materials. Potyrailo and Morris [65] employed a 13.56-MHz RFID tag sensor coated with a solid-polymer-electrolyte sensor film to determine resistance and identify vapor. When the RFID tag was coated with the sensor film, the localization accuracy of the RFID tag was lower. Potyrailo et al. [66] performed principal component analysis on the measurement parameters of adjustment tag sensors to prevent a decrease in the localization accuracy of the sensors. The results revealed that the localization function and signal strength of the RFID tag were most stable in a dry environment. By contrast, in environments with more than $45 \%$ humidity, the signal strength of the RFID tag was unstable. Potyrailo et al. [67] developed a new RFID tag sensor capable of resisting electromagnetic interference. In another study, Potyrailo et al. [68] employed RFID tag sensors in pharmaceutical manufacturing. They revealed that the temperature and electrical conductivity of a solution influenced the drug identification performance of the sensor. Potyrailo et al. [69] introduced RFID tag sensors to roll-to-roll technology manufacturing for measuring resonance impedance and then performed various tests with different spectrum characteristics and vapor densities. Subsequently, they applied RFID tags to test the freshness of food and monitor its safety. Kassal et al. [70] employed a low-power RFID tag sensor with an automatically measurable internal electrode potential to transmit recorded data through RFID to a reader. Steinberg et al. [71] combined RFID with photometer tags for short-distance wireless transmission.

They reported that circular antenna passive RFID tags can store collected sensor data. Grosinger et al. [72] discovered that the antenna of RFID tag sensors achieved a $92 \%$ transmission rate at $915 \mathrm{MHz}$. Caccami and Marrocco [73] reported that in linear and nonlinear regimes, electromagnetic models influence an antenna's resistance. Therefore, the researchers proposed the necessity of self-tuning RFID tags to prevent drops in the transmission efficiency of sensors. The path can be observed to split, with one subpath being led by Zannas et al. [74], who developed self-tuning UHF RFID sensor antennas, and the other being led by Hillie et al. [75], who placed tag sensors in electrolyte solutions to change 
their capacitance, enabling self-tuning and resistance adjustment. These six papers revealed Potyrailo to be a main contributor in this path. From 2007 to 2014, RFID was applied for the identification of chemical substances, drugs, and gasses; for $\mathrm{pH}$ measurements; and in optical and printing applications. By 2018, RFID tag sensors had self-tuning functions and were capable of adjusting to environmental changes. This was crucial to the research and development of sensors.

\subsubsection{Group 6: Localization Algorithms (Period: 2003-2019)}

The center path in Figure 5 consists of papers on the optimization of RFID localization algorithms. Han et al. [76] proposed a triangular RFID tag layout with which robot localization and orientation could be rapidly and accurately performed. Gueaieb and Miah [77] presented a localization system established by placing RFID tags in a threedimensional space; the system then defines the lines linking the tags on the ground as movement paths for the robot. Antennas can effectively read the relative localization errors within a given region. To reduce localization errors, Park and Hashimoto [78] proposed an algorithm that employs passive RFID. By using trigonometric functions and the Cartesian coordinates of UWB RFID tags, the algorithm can precisely estimate a robot's location and orientation. Saab and Nakad [79] proposed an indoor positioning system that employs the Kalman filter to calculate the location of a reader. Digiampaolo and Martinelli [80] used the Kalman filter to combine RFID reader data with the mileage data of indoor autonomous vehicles; they achieved a mean localization error of approximately $10 \mathrm{~cm}$. The same researchers also presented a system capable of achieving a mean localization error of $4 \mathrm{~cm}$ in which only two tags are used [64].

Zhang et al. [13] observed how interactions between tags influence the k-nearest neighbor (k-NN) algorithm in calculating the radiation orientation of tag antennas and discovered that the revised k-NN algorithm achieves a small mean localization error. Scherhaeuf et al. [81] developed a localization measurement system comprising conventional UHF RFID tags and commercial RFID readers. The system makes accurate localization measurements in an $8.75-\mathrm{m}^{2}$ office, with the maximum estimation error being $0.032 \mathrm{~m}$. According to the development trajectory indicated by the present analysis, the k-NN algorithm is the most popular algorithm used in this field. However, estimation of the k-value is a crucial problem. Additionally, because multiple methods are available for RSSI analysis, further improvement of the localization accuracy is difficult. Zhao et al. [82] proposed a novel similarity analysis-based indoor localization algorithm for indoor localization to optimize localization systems. Shi et al. [83] established a new network planning model for determining the locations of a fixed number of readers; use of this model results in the minimal number of localization errors and amount of interference. Shi et al. [84] proposed applying IKULDAS, an improved k-NN-based indoor localization algorithm, in localization and orientation systems to reduce the operation time and improve the precision of localization. Al-Jarrah et al. [85] employed an error concealment algorithm to improve the accuracy of location estimations. By using the location of adjacent tags, the algorithm estimates the error in the estimation; this approach achieves $26.5 \%$ higher estimation accuracy than benchmark estimators do. 


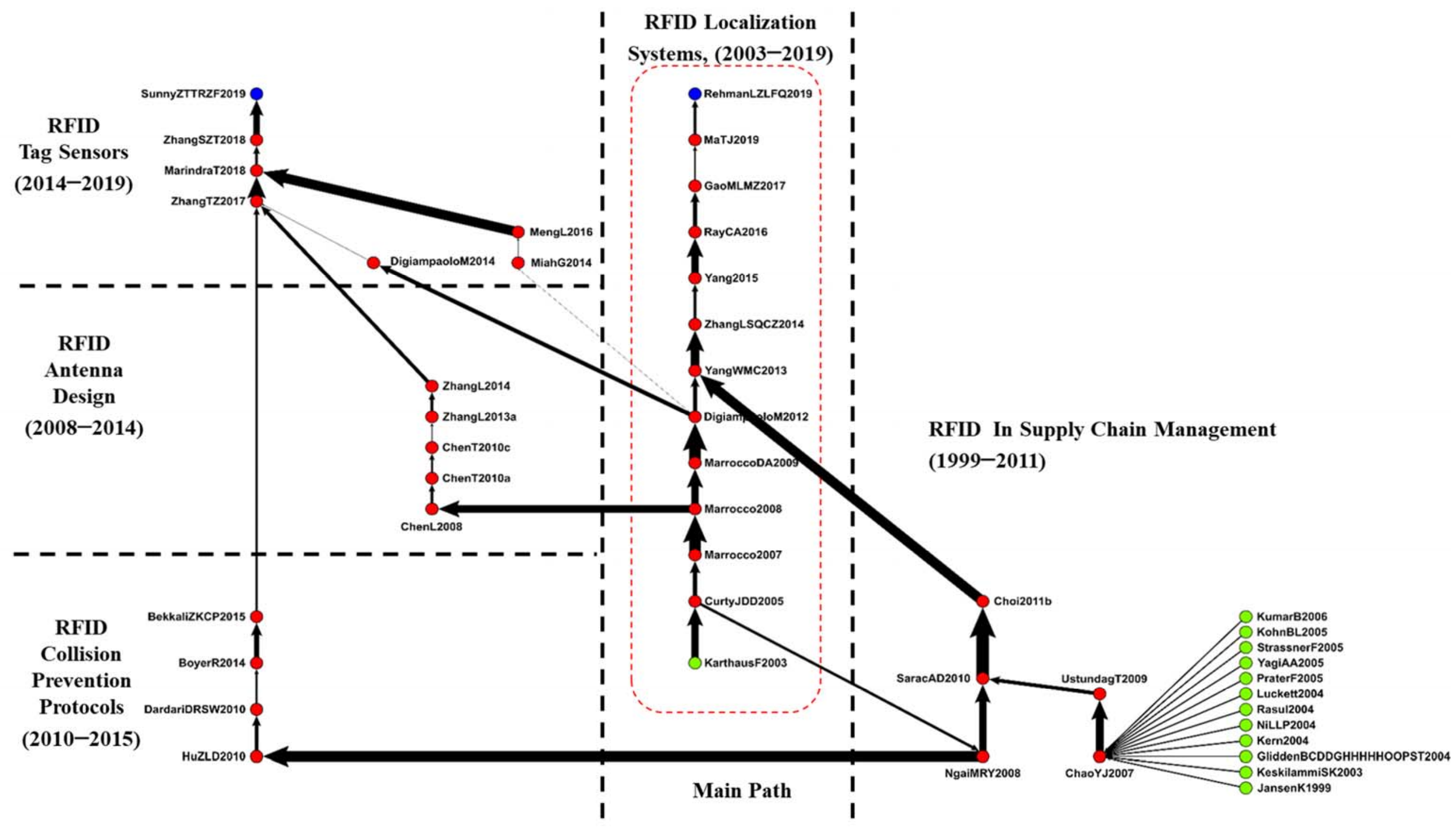

Figure 5. The development trajectory of academic papers on RFID research. 
In this study, the relationships between the six main clusters and the main path are illustrated in Figure 5. These themes were radio frequency identification applications in supply chain management, antenna design, collision prevention protocols, tag sensors, and localization systems in the main path. After using the specified branches analysis of keywords, it is possible to clearly understand the relationship between grouping and keyroute main path. The results breakthrough that radio frequency identification applications in privacy and safety only appear on the key-route main path as shown in Figure 6.

\subsection{Technical Analysis of Text and Data as Well as Cluster Development Trajectory}

The development trajectory of the research reveals that manufacturing applications have been the most influential RFID applications. To understand the development of RFID technology in other fields, we clustered the citation networks and performed word cloud analysis on the paper titles in each cluster. Papers with higher citation counts in each cluster were referenced to determine the research theme of each group. We use the edge-betweenness clustering technique to identify the research themes of the RFID literature. The result consists of six groups with the number of papers from 224 to 705 along with many small groups that published less than 224 papers. Through the analysis, the results are twenty clusters, and six clusters with citation counts of more than 200 were obtained. Figure 7 lists the research theme, number of papers, key words and phrases, and word cloud analysis results for each cluster. In the word cloud analysis, statistical analysis was performed on the key words and phrases in the titles and content of papers in each cluster, and the results are presented as word clouds. After the papers collected from the WOS database were compiled into six clusters, cluster analysis was performed to determine the six main research themes. These themes were RFID applications in supply chain management, antenna design, collision prevention protocols, tag sensors, privacy and safety, and localization systems, with the clusters consisting of 705, 692, 406, 334, 300, and 224 papers, respectively. With the exception of privacy and safety applications, the RFID applications in these topics were discussed in Section 3.3.

\section{Discussion}

\subsection{Development Trajectory of RFID and Clusters}

In addition, relatively special applications were found in the clusters, i.e., RFID applications in privacy and safety, as recommendations for scholars to study the direction of RFID technology applications. RFID applications in privacy and safety are the key-route main path of RFID applications, indicating that this research topic is influential, but the relevant academic literature appears in the global main path. In this study, the overall development direction of studies on RFID was determined through main path analysis of the critical path. To guarantee consistency between the cluster and development direction and to evaluate and analyze the consistency between a cluster and its corresponding cluster, a technical analysis of text and data as well as a key-route main path analysis of the critical path were used to divide the citation network into various clusters.

First, this study analyzed the development path for the field of RFID. Academic development generally occurs along multiple channels. According to the paths developed through the key-route main path analysis of the critical path, one main paper were source points leading to one branch (Figure 6). The branch, which included the one connecting line on the yellow dotted frame, contained 33 studies of RFID (Jules, 2006). Subsequently, key-route main path analyses of the critical paths of the clusters were performed. In the main path of a critical path, each node represents a study, and connecting lines represent citation relationships between studies. 


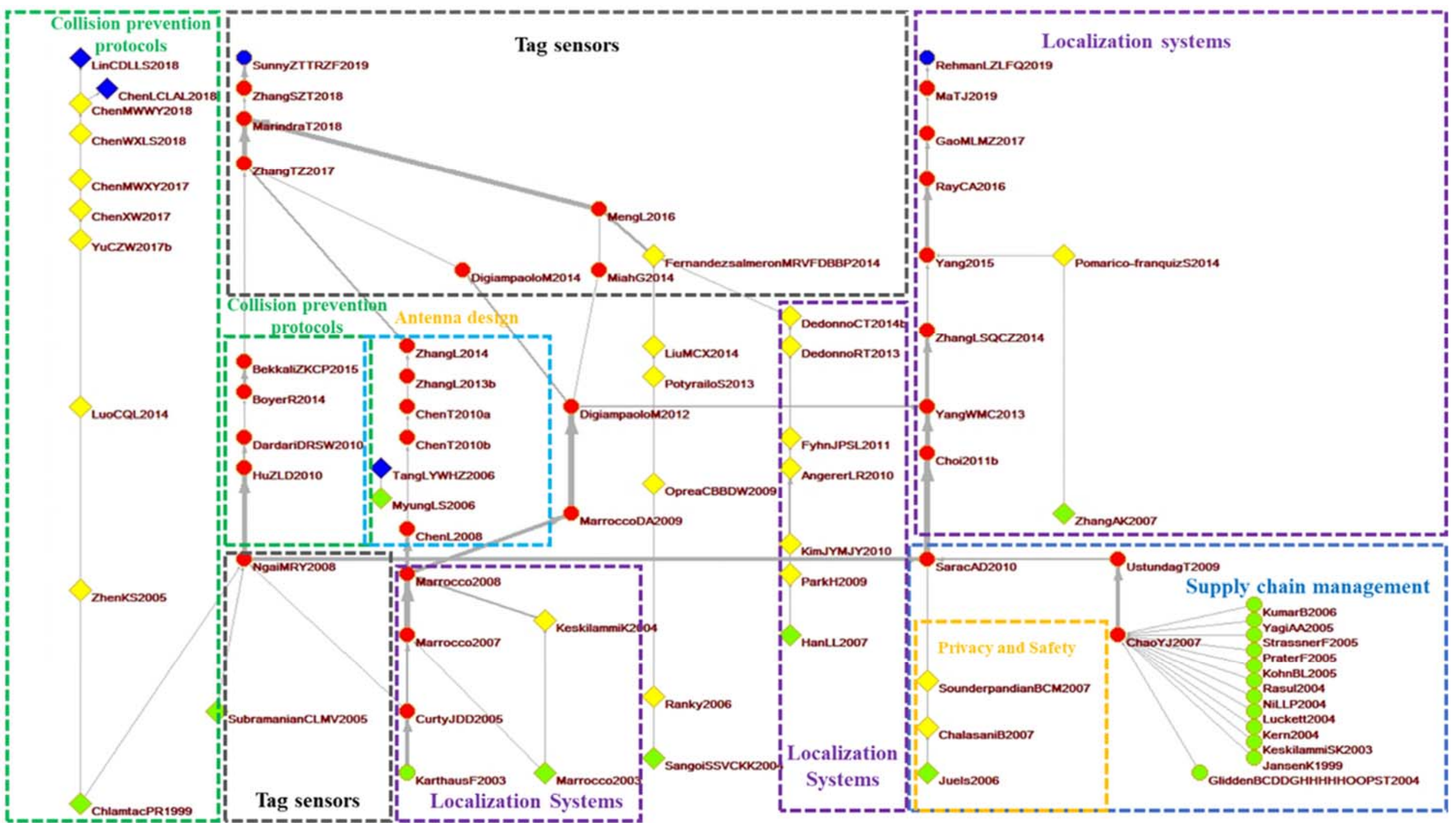

Figure 6. The development trajectory of academic papers on RFID research. 


\begin{tabular}{|c|c|c|c|c|c|c|}
\hline Theme & $\begin{array}{l}\text { RFID Applications in } \\
\text { Supply Chain Managemen }\end{array}$ & $\begin{array}{l}\text { RFID Applications in } \\
\text { Antenna Design }\end{array}$ & $\begin{array}{l}\text { RFID Applications in } \\
\text { Collision Prevention } \\
\text { Protocols }\end{array}$ & $\begin{array}{l}\text { RFID Applications in Tag } \\
\text { Sensors }\end{array}$ & $\begin{array}{l}\text { FID Applications in } \\
\text { Privacy and Safety }\end{array}$ & $\begin{array}{l}\text { FID Applications in } \\
\text { Localization Systems }\end{array}$ \\
\hline Papers & 705 & 692 & 406 & 334 & 300 & 224 \\
\hline Keywards & $\begin{array}{l}\text { supply }(0.212), \text { chain }(0.187) \text {, } \\
\text { management }(0.144), \\
\text { food }(0.033), \text { Design }(0.031) \text {, } \\
\text { impact }(0.031)\end{array}$ & $\begin{array}{l}\text { Antemna }(0.331) \text {, } \\
\text { Tag }(0.340), \text { Design }(0.065) \text {, } \\
\text { Metallic }(0.060) \text {, } \\
\text { Passive }(0.027) \text {, } \\
\text { Wireless }(0.014), \\
\text { Planar }(0.014)\end{array}$ & $\begin{array}{l}\text { Algorithun }(0.140), \\
\text { Protocol }(0.134), \\
\text { anti-collision }(0.167) \text {, } \\
\text { Adaptive }(0.023), \\
\text { Optimal }(0.023)\end{array}$ & $\begin{array}{l}\text { Sensor }(0.255), \\
\text { Passive }(0.108), \text { Tag }(0.092), \\
\text { System }(0.030), \text { Backscatter }(0 \\
.024), \text { Temperature }(0.024), \\
\text { Technology }(0.020), \\
\text { Flexible }(0.020), \\
\text { Hunidity }(0.020)\end{array}$ & $\begin{array}{l}\text { Authentication }(0.325) \text {, } \\
\text { Security }(0.258), \text { Mutual } \\
(0.022), \text { Privacy }(0.069), \\
\text { lightweight }(0.029), \\
\text { tags }(0.029)\end{array}$ & $\begin{array}{l}\text { Localization }(0.258) \text {, } \\
\text { Passive }(0.120) \text {, } \\
\text { Indoor }(0.135), \\
\text { System }(0.113), \text { Tags }(0.135) \text {, } \\
\text { Algorithn }(0.074) \text {, } \\
\text { Tracking }(0.037) \text {, }\end{array}$ \\
\hline
\end{tabular}

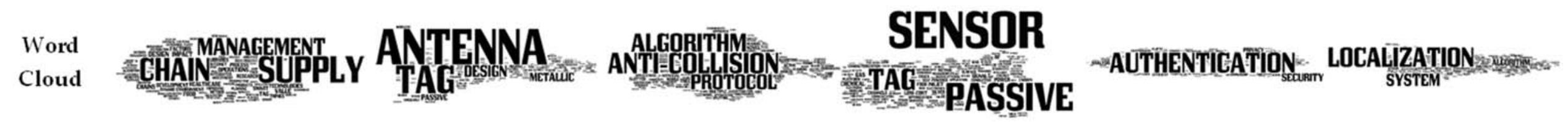

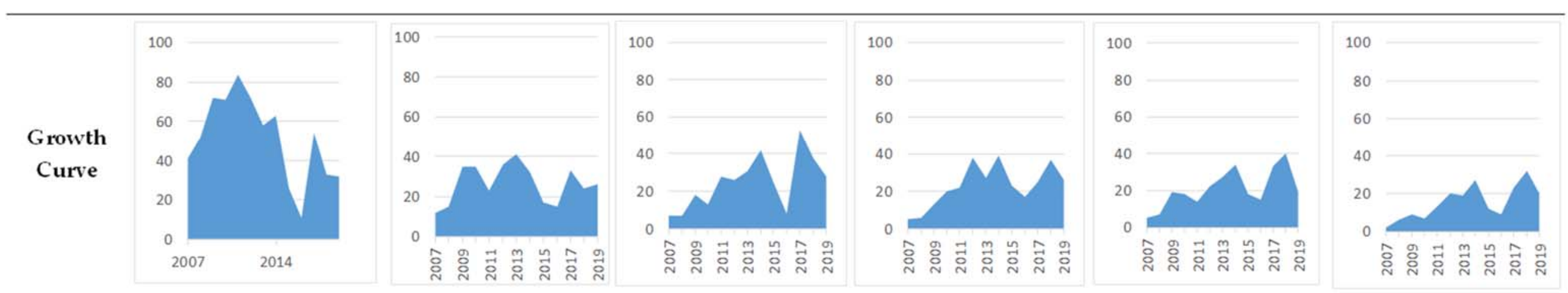

Figure 7. Themes, number of papers, keywords, word cloud and growth curve of each group. 
Group 5: Privacy and Safety (Period: 2006-2018)

Juels [86] examined the privacy protection and comprehensive protection measures for RFID systems proposed by scientists and considered the social and technical contexts of this research. Huang et al. [87] researched the application of RFID protocols in tag readers for mutual identity authentication. Ning et al. [88] proposed the key array authentication protocol; a logistical, safety, and performance analysis of the protocol determined it to be reliable in RFID systems. Chou [89] proposed an RFID mutual authentication protocol based on elliptic curve cryptography and discussed its application in security problems. Subsequently, Farash [90] described the shortcomings of the protocol proposed by Chou, indicating that the protocol could not ensure tag privacy, forwarding secrecy, identity authentication, or server authentication and was unable to protect against simulated, tag cloning, and location-tracking attacks. He and Zeadally proposed an RFID identity authentication solution for medical environments [91]. Anandhi et al. [92] employed the elliptic curve Diffie-Hellman protocol to generate a shared key and encrypt transmitted data. To solve identity authentication and privacy protection problems in campus security monitoring, Zheng et al. [93] employed RFID to improve campus security authentication and established a system for mutual authentication among tags, readers, and the backend database. This system effectively blocks simulated, location-tracking, synchronized, eavesdropping, and denial-of-service attacks. Lee et al. [94] calculated the offloading capacity of IoT devices, analyzed the power consumption of different components in a mobile RFID system, and proposed a model for reducing the power consumption of similar systems. In IoT applications, the power supply and computational capability of sensors are limited. Therefore, future studies can focus on reducing power consumption.

One of the major concerns in many applications is the security and privacy of data. The main factor is the fast expansion and Internet diversification in the IoT and related services, such as used protocols and mutual authentication protocol. Papers have introduced the leading wireless identification technologies, traced their evolution, explained their purpose, assessed their privacy risks, and evaluated proposed solutions. The results show the cluster developments of academic papers on RFID and the connections among the six research clusters. Figure 7 presents the relationships among the six major groups of RFID research topics and the main path, where only papers connected to said six groups are labeled.

\subsection{Emerging Area or Potential Opportunity in Other Applications}

In the literature review, more than 18 papers on RFID applications in nonmanufacturing industries were collected, and four other clusters of research themes were discovered (Table 2). These clusters, listed in order of descending frequency, represent RFID applications in construction material management, beehive model experiments, erratic tracking experiments, and aquaculture. Because few studies have been published in these fields, not all the papers were present in the main path. Additionally, papers conducting literature reviews in these fields only reported the development progress of specific fields. Although these papers contributed and added value to these fields, the present study only considered influential papers and research fields with many publications (Table 2).

The literature review revealed that in construction material management, RFID applications have provided support in material planning, procurement, storage, processing, distribution, land use, construction monitoring, and progress reporting, thereby enhancing construction efficiency and preventing over-expenditure and construction delays. In beehive model experiments, RFID has been used to obtain a four-dimensional behavioral model of bees entering and exiting a beehive. In papers discussing the mutual interactions between wave activity and rocky coasts, RFID tags and localization systems have been employed to accurately measure the displacement of ocean sediments. The fewest papers have been published on RFID applications in aquaculture because the use of RFID in feeding management is currently a novel application.

RFID technology can be used to automatically determine the health and vitality of livestock, after which their feeding can be altered to achieve the optimal allocation of feed 
resources. In other clusters, a few papers have been published on RFID applications in feeding management. The trend analysis revealed that in research on electronic antennas and tag technologies, RFID applications are no longer the overall development trend; RFID has been widely applied in various new applications.

Table 2. Research themes ranked seventh to tenth.

\begin{tabular}{ccccc}
\hline Cluster & Research Theme & Published Year & Number of Papers & Is the Maturity Period? (Yes/No) \\
\hline 7 & Construction material management & $1995-2019$ & 145 & No, it is the growth period in 2019. \\
8 & Beehive model experimentation & $2002-2019$ & 66 & No, it is mature in 2017. \\
9 & Erratic tracking experimentation & $2006-2019$ & 23 & Yes, it is mature in 2019. \\
10 & Aquaculture & $2011-2019$ & 18 & No, it is the growth period in 2019. \\
\hline
\end{tabular}

\subsection{Pattern of Growth and Studying Feasibility in the Future}

The Loglet Lab4 software (http://phe.rockefeller.edu/LogletLab4, accessed on 26 February 2018) was used to determine the growth period, limit, and turning points. The dashed line in Figure 8 represents the cumulative number of published papers, whereas the solid line and dots indicate the actual cumulative number of published papers. The curve indicates a maturity period after 2020, when the maximum cumulative number of published papers would be approximately 4000 . In this study, the results further indicated that the RFID topic remains in a pattern of growth, more valuable, the corresponding curve is near the inflection point, and the topic is more than 10 years away from maturity.

\section{Growth of RFID Article}

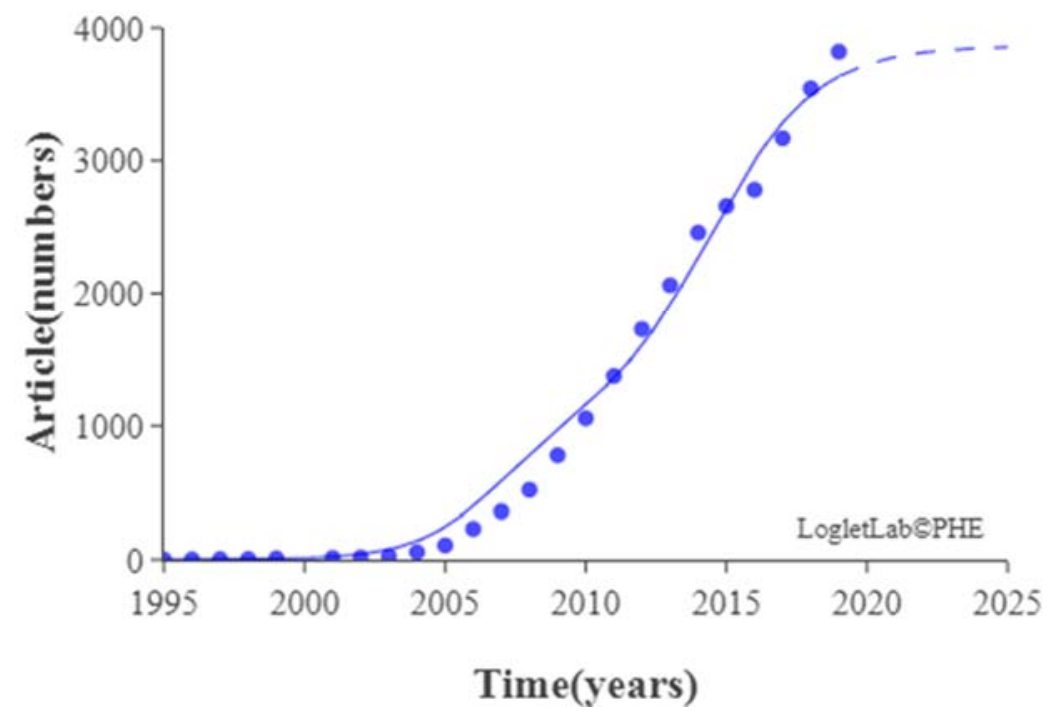

Figure 8. Growth curve of the numbers of papers.

\section{Conclusions}

The present study determined the influential articles, authors, and journals related to the main research topics and the development directions of the field of RFID. Moreover, a citation analysis and a key-route main path analysis of critical papers were conducted to identify the core research in studies of RFID. The growth trajectory of academic papers was described to provide a deeper understanding of the development trends of research into RFID.

The present study combined main path, clustering, and text mining analyses to explore the overall development trajectory of the RFID-related literature. The main path analysis revealed that RFID applications have mainly focused on manufacturing. Key-route main path analyses of the critical paths were performed to analyze the research topics emphasized in the clusters. The overall development track and the connections between clusters 
were explored through six topics, namely in supply chain management, antenna design, collision prevention protocols, privacy and safety, tag sensors, and localization systems. In the literature review, more than papers on RFID applications in nonmanufacturing industries were collected, and four other clusters of research themes were discovered the technology has been successfully applied in construction and aquaculture. The following five conclusions were drawn:

1. RFID applications in supply chain management: Such applications enhance the operating time, reduce management costs and perceived risk, and eliminate idle time. Thus, in these RFID applications, multiple targets can be simultaneously and rapidly identified, and information storage advantages more favorable than those offered barcodes are provided.

2. RFID applications in antenna design: The aforementioned authors suggested that researchers should focus on RFID localization algorithms, which have continually been developed. Overall, various RFID-based solutions have been proposed for improving the properties of antennas.

3. RFID applications in collision prevention protocols: RFID technology has been applied to collision prevention because signal collision reduces the signal's communication capacity, lowers system performance, and consumes considerable power.

4. RFID applications in localization systems: By using the locations of adjacent tags, the corresponding algorithm can try and error estimation, thereby leading to higher estimation accuracy.

5. RFID applications in tag sensors: RFID tag sensor technology has self-tuning functionality and can thus adjust to environmental changes.

In addition, relatively special applications were found in the clusters-applications in construction material management, applications in beehive model experiments, aquaculture, and erratic tracking experimentation. As a result of the radio frequency identification applications combine with the emerging technology, in construction material management and aquaculture are currently growing topics. The following are four conclusions:

1. RFID applications in construction material management: By implementing RFID technology, a construction company can overcome many of the issues and frustrations of managing and tracking materials. In the past five years, scholars have gained a clear understanding that Internet of Things (IoT) and emerging technologies have begun to be applied to material management systems in the literature.

- A lot of research introduces an IoT-based shop floor material management system that can capture dynamic data in real-time and effectively synthesize it along the supply chain. The proposed system can lead to cost reduction, improved efficiency, and improved compliance with just-in-time (JIT) inventory management principles.

- Due to the application of many new technology such as smart manufacturing, artificial intelligence, and RFID application have been presented the growing trend in past 3 years.

2. RFID applications in beehive model experiments: Until early 2000, direct observations and video recording of the foraging activity of social bees were the predominant techniques for studying bees' foraging behavior, and paint marks or labels were used to distinguish individuals. Although these techniques are still used, RFID technology has been used for bee monitoring and can automatically count the inbound and outbound movements of bees from the nest as well as recognize individuals.

- $\quad$ Although these techniques are still used, RFID technology has been used for bee monitoring and can automatically count the inbound and outbound movements of bees from the nest and perform individual recognition.

- In the literature over three years, new technologies have discovered a method for mining combined data from in-hive sensors, weather, and apiary inspections to forecast the health status of honey bee colonies. 
3. RFID applications in erratic tracking experimentation: These applications enable investigation of the interactions between wave activity and rocky coasts. RFID tags and localization systems have been employed to accurately measure the displacement of ocean sediments. Scholars have discovered that there are new trends applications in experimental environments such as monitoring low-energy high-temperature exposure 3D tags sensor, by deep learning principles as it outperforms traditional machine learning models to solve any tasks especially.

4. RFID applications in aquaculture: Convenient and automated systems for managing feeding on aquaculture farms are necessary to reduce costs and labor and for fish health management. In an RFID-based feeding system, a mass analyzer can identify the movement of feed and input the appropriate amount of feed into fish tanks. The input amount of feed is estimated by considering the change in weight detected by the mass analyzer. In addition, installing RFID readers, antennas, and tags is suggested for discriminating the feeding tank from a nearby tank. In the past three years, scholars have combined IoT and CIA technology to improve the performance of aquaculture operations. The researchers found that aquaculture is a potential emerging application, and the application is growing at present for the followings reason:

- The advent of Cloud computing, Internet of Things, and Artificial Intelligence (CIA) has expanded numerous possibilities for applying and integrating information technology in all works of life.

- In addition, the application of CIA in the aquaculture value chain to ensure effectiveness in traceability, feeding, disease detection, growth prediction, environmental monitoring, market information, and others is key to increasing aquaculture productivity and sustainability.

- Thus, the future of aquaculture operations with less human labor, effective maintenance, and resource utilization largely depend on innovative technologies at present.

The present study adopted the key-route main cluster path analysis in 3820 highly relevant papers of radio frequency identification to identify the paths of clusters and to develop their overall trajectories. In addition, relatively special applications were found in the clusters, i.e., radio frequency identification applications in privacy and safety, both databases (Scopus and Web of Science) recommend scholars study the direction of radio frequency identification technology applications in followings:

1. RFID applications in privacy and safety is the key-route main path of RFID applications, indicating that this research topic is valuable and influential, but the relevant academic literature does not appears in the global main path. Most RFID system try to design a high-level security, but not all the RFID applications need it. During this research, it is growing, with management of the complexity in security models and protocols.

2. By growth curve, the results further indicated that the RFID topic remains in a pattern of growth, and the topic is more than 10 years away from maturity. The results of the study confirm the radio frequency identification is a valuable topic. When RFID applications integrate emerging technologies such as IoT, smart manufacturing and artificial intelligence, it is an important hybrid topic.

3. The next researcher's recommendations collected and used information from the Scopus and Web of Science databases. Cesar et al. [95] retrieved the data from the both databases, and performed the query using the string ("Radio frequency identification" OR "Radio-frequency identification" OR "RFID") to obtain the data in both database.

First, our text mining analysis of academic papers enabled us to predict the development trajectory of RFID-based research. Subsequently, six research topics that developed within the past 4 years in the academic research field of radio frequency identification were identified and inferred to be possible future directions for technical portfolios. Academics interested in radio frequency identification technological development may reference these 
topics for planning future research and development strategies. This review can help motivate and inform studies in this field.

Based on the literature data collected RFID-related papers from the WOS database and conducted a literature review. A limitation of this study was that this paper may miss some important and related papers because the database used in this paper is only the WOS. Secondly, papers about these domains are not full and the results of the main path analysis may change when the number of papers changes. Finally, though review papers play an important role in the development of these domains, it remains controversial whether to remove review papers for main path analysis. Moreover, the results of the study can help future researchers to define their research directions, understand the development trajectory of the field, technology forecasting, and patterns mined can be utilized by the business.

Author Contributions: Final approval of the manuscript: all authors. Conceptualization, W.-H.S. and J.-J.W.; methodology, L.Y.Y.L.; software, W.-H.S.; validation, W.-H.S., K.-Y.C. and L.Y.Y.L.; formal analysis, W.-H.S.; investigation, J.-J.W.; resources, L.Y.Y.L.; data curation, W.-H.S.; writing-original draft preparation, J.-J.W.; writing-review and editing, W.-H.S.; visualization, W.-H.S. and L.Y.Y.L.; project administration, W.-H.S. All authors have read and agreed to the published version of the manuscript.

Funding: This research received no external funding.

Institutional Review Board Statement: The study was conducted according to the guidelines of the Declaration of Helsinki and approved by the Institutional Review Board. Ethical review and approval were waived for this study.

Informed Consent Statement: Not applicable.

Data Availability Statement: Data sharing is not applicable to this article.

Conflicts of Interest: The authors declare no conflict of interest.

\section{References}

1. ResearchGate. Technologies for Industry 4.0. Available online: https:/ /www.researchgate.net/figure/Technologies-for-industry40_fig1_319944621 (accessed on 19 October 2018).

2. Convergence Training. RFID Applications. Available online: https://www.convergencetraining.com/rfid-applications.html (accessed on 3 December 2019).

3. ARC Advisory Group. ARC Advisory Group Announces New RFID in Manufacturing Market Research. Available online: https:// www.arcweb.com/press/arc-advisory-group-announces-new-rfid-manufacturing-market-research (accessed on 2 August 2016).

4. IRISS. Increasing Resilience in Surveillance Societies. Available online: http://irissproject.eu/wp-content/uploads/2013/06/ IRISS-D1-MASTER-DOCUMENT-10-June-2013.pdf (accessed on 10 June 2013).

5. DTechEx. RFID Market by Product (Tags, Reader, and Software), Wafer Size, Working (Passive, and Active), Frequency (Low, High, Ultra-High), Applications, Form Factor (Button, Card, Electronic Housing, Implants), Label Type, and Region-Global Forecast to 2023. Available online: https:/ / kknews.cc/tech/bz8kbv9.html (accessed on 12 November 2019).

6. iFair. RFID Technology and Theory. Available online: https://www.ifair.com.tw/product (accessed on 1 January 2018).

7. Karthaus, U.; Fischer, M. Fully integrated passive UHF RFID transponder IC with 16.7- $\mu$ W minimum RF input power. IEEE J. Solid State Circuits 2003, 308, 1602-1608. [CrossRef]

8. Curty, J.P.; Joehl, N.; Dehollain, C.; Declercq, M.J. Remotely powered addressable UHF RFID integrated system. IEEE J. Solid State Circuits 2005, 40, 2193-2202. [CrossRef]

9. Marrocco, G. RFID antennas for the UHF remote monitoring of human subjects. IEEE Trans. Antennas Propag. 2007, 55, 1862-1870. [CrossRef]

10. Marrocco, G. The art of UHF RFID antenna design: Impedance-matching and size-reduction techniques. IEEE Antennas Propag. Mag. 2008, 50, 66-79. [CrossRef]

11. Marrocco, G.; Di Giampaolo, E.; Aliberti, R. Estimation of UHF RFID reading regions in real environments. IEEE Antennas Propag. Mag. 2009, 51, 44-57. [CrossRef]

12. Yang, P.; Wu, W.; Moniri, M.; Chibelushi, C.C. Efficient object localization using sparsely distributed passive RFID tags. IEEE Trans. Ind. Electron. 2012, 60, 5914-5924. [CrossRef]

13. Zhang, Z.; Lu, Z.; Saakian, V.; Qin, X.; Chen, Q.; Zheng, L.R. Item-level indoor localization with passive UHF RFID based on tag interaction analysis. IEEE Trans. Ind. Electron. 2013, 61, 2122-2135. [CrossRef]

14. Yang, P. RLS-INVES: A general experimental investigation strategy for high accuracy and precision in passive RFID location systems. IEEE Internet Things J. 2014, 2159-2167. 
15. Ray, B.R.; Chowdhury, M.U.; Abawajy, J.H. Secure object tracking protocol for the Internet of things. IEEE Internet Things J. 2016, 3, 544-553. [CrossRef]

16. Gao, Z.; Ma, Y.; Liu, K.; Miao, X.; Zhao, Y. An indoor multi-tag cooperative localization algorithm based on NMDS for RFID. IEEE Sens. J. 2017, 17, 2120-2128. [CrossRef]

17. Marindra, A.M.J.; Tian, G.Y. Chipless RFID sensor tag for metal crack detection and characterization. IEEE Trans. Microw. Theory Technol. 2018, 66, 2452-2462. [CrossRef]

18. Ur Rehman, S.; Liu, R.; Zhang, H.; Liang, G.; Fu, Y.; Qayoom, A. Localization of moving objects based on RFID tag array and laser ranging information. Electronics 2019, 8, 887. [CrossRef]

19. Liu, J.S.; Lu, L.Y.; Lin, L. Analyzing Technological Trajectory Via Patent Citation Network: An Application to Radio Frequency Identification Technology (RFID). Master's Thesis, Yuan Ze University, Taoyuan City, Taiwan, 2011.

20. Hummon, N.P.; Dereian, P. Connectivity in a citation network: The development of DNA theory. Soc. Netw. 1989, 11, 39-63. [CrossRef]

21. Batagelj, V. Efficient algorithms for citation network analysis. arXiv 2003, arXiv:cs/0309023.

22. Liu, J.S.; Lu, L.Y. An integrated approach for main path analysis: Development of the Hirsch index as an example. J. Am. Soc. Inf. Sci. Technol. 2012, 63, 528-542. [CrossRef]

23. Liu, J.S.; Lu, L.Y.; Lu, W.M.; Lin, B.J. Data envelopment analysis 1978-2010: A citation-based literature survey. Omega 2013, 41, 3-15. [CrossRef]

24. Ho, J.C.; Saw, E.C.; Lu, L.Y.; Liu, J.S. Technological barriers and research trends in fuel cell technologies: A citation network analysis. Technol. Forecast. Soc. Chang. 2014, 82, 66-79. [CrossRef]

25. Liu, J.S.; Lu, L.Y.; Lu, W.M. Research fronts in data envelopment analysis. Omega 2016, 58, 33-45. [CrossRef]

26. Chuang, T.C.; Liu, J.S.; Lu, L.Y.; Tseng, F.M.; Lee, Y.; Chang, C.T. The main paths of eTourism: Trends of managing tourism through Internet. Asia Pac. J. Tour. Res. 2017, 22, 213-231. [CrossRef]

27. Yan, J.; Tseng, F.M.; Lu, L.Y. Developmental trajectories of new energy vehicle research in economic management: Main path analysis. Technol. Forecast. Soc. Chang. 2018, 137, 168-181. [CrossRef]

28. Su, W.H.; Chen, K.Y.; Lu, L.Y.; Wang, J.J. Identification of technology diffusion by citation and main paths analysis: The possibility of measuring open innovation. Open Innov. Technol. Mark. Complex. 2021, 7, 104. [CrossRef]

29. Fontana, R.; Nuvolari, A.; Verspagen, B. Mapping technological trajectories as patent citation networks: An application to data communication standards. J. Manag. Stud. 2009, 49, 1351-1374. [CrossRef]

30. Consoli, D.; Mina, A. An evolutionary perspective on health innovation system. J. Evol. Econ. 2009, 19, 297-319. [CrossRef]

31. Liu, J.S.; Lu, L.Y.; Hsieh, C.H.; Chen, S.J. Developmental trajectory and trend of the broad virtual reality. J. Manag. Syst. 2019, 26, 427-449.

32. Hirsch, J.E. An index to quantify an individual's scientific research output. Proc. Natl. Acad. Sci. USA 2005, 102, 16569-16572. [CrossRef] [PubMed]

33. Egghe, L. Theory and practise of the g-index. Scientometrics 2006, 69, 131-152. [CrossRef]

34. Girvan, M.; Newman, M.E. Community structure in social and biological networks. Proc. Natl. Acad. Sci. USA 2002, 99, $7821-7827$. [CrossRef]

35. Newman, M.E.J.; Girvan, M. Finding and evaluating community structure in networks. Phys. Rev. E 2004, 69, 026113. [CrossRef]

36. Newman, M.E.J. Fast algorithm for detecting community structure in networks. Phys. Rev. E 2004, 69, 066133. [CrossRef]

37. Newman, M.E.J. Modularity and community structure in networks. Proc. Natl. Acad. Sci. USA 2006, 103, 8577-8582. [CrossRef]

38. Wordle. Web of Science. Available online: http:/ / www.wordle.net/ (accessed on 12 October 2019).

39. Verhulst, P.F. Recherches mathématiques sur La Loi D’Accroissement de la population. Nouv. Mémoires Académie R. Sci. Belles-Lett. Brux. 1845, 18, 1-45.

40. Chao, C.C.; Yang, J.M.; Jen, W.Y. Determining technology trends and forecasts of RFID by a historical review and bibliometric analysis from 1991 to 2005. Technovation 2007, 27, 268-279. [CrossRef]

41. Jansen, R.; Krabs, A. Automatic identification in packaging-Radio frequency identification in multiway systems. Packag. Technol. Sci. 1999, 12, 229-234. [CrossRef]

42. Ustundag, A.; Tanyas, M. The impacts of radio frequency identification (RFID) technology on supply chain costs. Transp. Res. E Logist. Transp. Rev. 2009, 45, 29-38. [CrossRef]

43. Chen, J.C.; Cheng, C.H.; Huang, P.B. Supply chain management with lean production and RFID application: A case study. Expert Syst. Appl. 2013, 40, 3389-3397. [CrossRef]

44. Vlachos, I.P. A hierarchical model of the impact of RFID practices on retail supply chain performance. Expert Syst. Appl. 2014, 41, 5-15. [CrossRef]

45. Ding, K.; Jiang, P.; Su, S. RFID-enabled social manufacturing system for inter-enterprise monitoring and dispatching of integrated production and transportation tasks. Robot. Comput. Integr. Manuf. 2018, 49, 120-133. [CrossRef]

46. Kang, Y.S.; Kim, H.; Lee, Y.H. Implementation of an RFID-based sequencing-error-proofing system for automotive manufacturing logistics. Appl. Sci. 2018, 8, 109. [CrossRef]

47. Hirvonen, M.; Pursula, P.; Jaakkola, K.; Laukkanen, K. Planar inverted-Fantenna for radio frequency identification. Electron. Lett. 2004, 40, 848-850. [CrossRef] 
48. Padhi, S.K.; Karmakar, N.C.; Law, C.L.; Aditya, S. A dual polarized aperture coupled circular patch antenna using a C-shaped coupling slot. IEEE Trans. Antennas Propag. 2003, 51, 3295-3298. [CrossRef]

49. Rao, K.S.; Nikitin, P.V.; Lam, S.F. Antenna design for UHF RFID tags: A review and a practical application. IEEE Trans. Antennas Propag. 2005, 53, 3870-3876. [CrossRef]

50. Chen, S.L.; Lin, K.H. A slim RFID tag antenna design for metallic object applications. IEEE Antennas Wirel. Propag. Lett. 2008, 7, 729-732. [CrossRef]

51. Yan, Y.; Ouyang, J.; Ma, X.; Wang, R.; Sharif, A. Circularly polarized RFID tag antenna design for metallic poles using characteristic mode analysis. IEEE Antennas Wirel. Propag. Lett. 2019, 18, 1327-1331. [CrossRef]

52. Hu, S.; Zhou, Y.; Law, C.L.; Dou, W. Study of a uniplanar monopole antenna for passive chipless UWB-RFID localization system. IEEE Trans. Antennas Propag. 2009, 58, 271-278.

53. Dardari, D.; d'Errico, R.; Roblin, C.; Sibille, A.; Win, M.Z. Ultrawide bandwidth RFID: The next generation? Proc. IEEE 2010, 98, 1570-1582. [CrossRef]

54. Cha, K.; Jagannathan, S.; Pommerenke, D. Adaptive power control protocol with hardware implementation for wireless sensor and RFID reader networks. IEEE Syst. J. 2007, 1, 145-159.

55. Kim, D.Y.; Yoon, H.G.; Jang, B.J.; Yook, J.G. Effects of reader-to-reader interference on the UHF RFID interrogation range. IEEE Trans. Ind. Electron. 2009, 56, 2337-2346.

56. Eom, J.B.; Yim, S.B.; Lee, T.J. An efficient reader anticollision algorithm in dense RFID networks with mobile RFID readers. IEEE Trans. Ind. Electron. 2009, 56, 2326-2336.

57. Gandino, F.; Ferrero, R.; Montrucchio, B.; Rebaudengo, M. Probabilistic DCS: An RFID reader-to-reader anti-collision protocol. J. Netw. Comput. Appl. 2011, 34, 821-832. [CrossRef]

58. Ferrero, R.; Gandino, F.; Montrucchio, B.; Rebaudengo, M. A fair and high throughput reader-to-reader anticollision protocol in dense RFID networks. IEEE Trans. Industr. Inform. 2011, 8, 697-706. [CrossRef]

59. Gandino, F.; Ferrero, R.; Montrucchio, B.; Rebaudengo, M. DCNS: An adaptable high throughput RFID reader-to-reader anticollision protocol. IEEE Trans. Parallel Distrib. Syst. 2012, 24, 893-905. [CrossRef]

60. Golsorkhtabaramiri, M.; Hosseinzadeh, M.; Reshadi, M.; Rahmani, A.M. A reader anti-collision protocol for RFID-enhanced wireless sensor networks. Wirel. Pers. Commun. 2015, 81, 893-905. [CrossRef]

61. Golsorkhtabaramiri, M.; Issazadehkojidi, N. A distance based RFID reader collision avoidance protocol for dense reader environments. Wirel. Pers. Commun. 2017, 95, 1781-1798. [CrossRef]

62. Golsorkhtabaramiri, M.; Issazadehkojidi, N.; Pouresfehani, N.; Mohammadialamoti, M.; Hosseinzadehsadati, S.M. Comparison of energy consumption for reader anti-collision protocols in dense RFID networks. Wirel. Netw. 2019, 25, 2393-2406. [CrossRef]

63. Liu, X.; Yang, Q.; Luo, J.; Ding, B.; Zhang, S. An energy-aware offloading framework for edge-augmented mobile RFID systems. IEEE Internet Things J. 2018, 6, 3994-4004. [CrossRef]

64. DiGiampaolo, E.; Martinelli, F. Mobile robot localization using the phase of passive UHF RFID signals. IEEE Trans. Ind. Electron. 2012, 61, 365-376. [CrossRef]

65. Potyrailo, R.A.; Morris, W.G. Multianalyte chemical identification and quantitation using a single radio frequency identification sensor. Anal. Chem. 2007, 79, 45-51. [CrossRef] [PubMed]

66. Potyrailo, R.A.; Mouquin, H.; Morris, W.G. Position-independent chemical quantitation with passive 13.56-MHz radio frequency identification (RFID) sensors. Talanta 2008, 75, 624-628. [CrossRef] [PubMed]

67. Potyrailo, R.A.; Morris, W.G.; Sivavec, T.; Tomlinson, H.W.; Klensmeden, S.; Lindh, K. RFID sensors based on ubiquitous passive 13.56-MHz RFID tags and complex impedance detection. Wirel. Commun. Mob. Comput. 2009, 9, 1318-1330. [CrossRef]

68. Potyrailo, R.A.; Wortley, T.; Surman, C.; Monk, D.; Morris, W.G.; Vincent, M.; Klensmeden, S. Passive multivariable temperature and conductivity RFID sensors for single-use biopharmaceutical manufacturing components. Biotechnol. Prog. 2011, 27, 875-884. [CrossRef]

69. Potyrailo, R.A.; Burns, A.; Surman, C.; Lee, D.J.; McGinniss, E. Multivariable passive RFID vapor sensors: Roll-to-roll fabrication on a flexible substrate. Analyst 2012, 137, 2777-2781. [CrossRef]

70. Kassal, P.; Steinberg, I.M.; Steinberg, M.D. Wireless smart tag with potentiometric input for ultra low-power chemical sensing. Sens. Actuators B Chem. 2013, 184, 254-259. [CrossRef]

71. Steinberg, M.D.; Kassal, P.; Tkalčec, B.; Steinberg, I.M. Miniaturised wireless smart tag for optical chemical analysis applications. Talanta 2014, 118, 375-381. [CrossRef]

72. Grosinger, J.; Görtschacher, L.; Bösch, W. Passive RFID sensor tag concept and prototype exploiting a full control of amplitude and phase of the tag signal. IEEE Trans. Microw. Theory Technol. 2016, 64, 4752-4762. [CrossRef]

73. Caccami, M.C.; Marrocco, G. Electromagnetic modeling of self-tuning RFID sensor antennas in linear and nonlinear regimes. IEEE Trans. Antennas Propag. 2018, 66, 2779-2787. [CrossRef]

74. Zannas, K.; El Matbouly, H.; Duroc, Y.; Tedjini, S. Self-tuning RFID tag: A new approach for temperature sensing. IEEE Trans. Microw. Theory Technol. 2018, 66, 5885-5893. [CrossRef]

75. Hillier, A.J.; Makarovaite, V.; Gourlay, C.W.; Holder, S.J.; Batchelor, J.C. A passive UHF RFID dielectric sensor for aqueous electrolytes. IEEE Sens. J. 2019, 19, 5389-5395. [CrossRef]

76. Han, S.; Lim, H.; Lee, J. An efficient localization scheme for a differential-driving mobile robot based on RFID system. IEEE Trans. Ind. Electron. 2007, 54, 3362-3369. [CrossRef] 
77. Gueaieb, W.; Miah, M.S. An intelligent mobile robot navigation technique using RFID technology. IEEE Trans. Instrum. Meas. 2008, 57, 1908-1917. [CrossRef]

78. Park, S.; Hashimoto, S. Autonomous mobile robot navigation using passive RFID in indoor environment. IEEE Trans. Ind. Electron. 2009, 56, 2366-2373. [CrossRef]

79. Saab, S.S.; Nakad, Z.S. A standalone RFID indoor positioning system using passive tags. IEEE Trans. Ind. Electron. 2010, 58, 1961-1970. [CrossRef]

80. DiGiampaolo, E.; Martinelli, F. A passive UHF-RFID system for the localization of an indoor autonomous vehicle. IEEE Trans. Ind. Electron. 2012, 59, 3961-3970. [CrossRef]

81. Scherhäufl, M.; Pichler, M.; Stelzer, A. UHF RFID localization based on phase evaluation of passive tag arrays. IEEE Trans. Instrum. Meas. 2014, 64, 913-922. [CrossRef]

82. Zhao, Y.; Liu, K.; Ma, Y.; Gao, Z.; Zang, Y.; Teng, J. Similarity analysis-based indoor localization algorithm with backscatter information of passive UHF RFID tags. IEEE Sens. J. 2016, 17, 185-193. [CrossRef]

83. Shi, W.; Guo, Y.; Yan, S.; Yu, Y.; Luo, P.; Li, J. Optimizing directional reader antennas deployment in UHF RFID localization system by using a MPCSO algorithm. IEEE Sens. J. 2018, 18, 5035-5048. [CrossRef]

84. Shi, W.; Du, J.; Cao, X.; Yu, Y.; Cao, Y.; Yan, S.; Ni, C. IKULDAS: An improved kNN-Based UHF RFID Indoor localization algorithm for directional radiation scenario. Sensors 2019, 19, 968. [CrossRef] [PubMed]

85. Al-Jarrah, M.A.; Al-Dweik, A.; Alsusa, E.; Damiani, E. RFID reader localization using hard decisions with error concealment. IEEE Sens. J. 2019, 19, 7534-7542. [CrossRef]

86. Juels, A. RFID security and privacy: A research survey. IEEE J. Sel. Areas Commun. 2006, 24, 381-394. [CrossRef]

87. Huang, Y.J.; Yuan, C.C.; Chen, M.K.; Lin, W.C.; Teng, H.C. Hardware implementation of RFID mutual authentication protocol. IEEE Trans. Ind. Electron. 2009, 57, 1573-1582. [CrossRef]

88. Ning, H.; Liu, H.; Mao, J.; Zhang, Y. Scalable and distributed key array authentication protocol in radio frequency identificationbased sensor systems. IET Commun. 2011, 5, 1755-1768. [CrossRef]

89. Chou, J.S. An efficient mutual authentication RFID scheme based on elliptic curve cryptography. J. Supercomput. 2014, 70, 75-94. [CrossRef]

90. Farash, M.S. Cryptanalysis and improvement of an efficient mutual authentication RFID scheme based on elliptic curve cryptography. J. Supercomput. 2014, 70, 987-1001. [CrossRef]

91. He, D.; Zeadally, S. An analysis of RFID authentication schemes for internet of things in healthcare environment using elliptic curve cryptography. IEEE Internet Things J. 2014, 2, 72-83. [CrossRef]

92. Anandhi, S.; Anitha, R.; Sureshkumar, V. An automatic RFID reader-to-reader delegation protocol for SCM in cloud computing environment. J. Supercomput. 2018, 74, 3148-3167. [CrossRef]

93. Zheng, L.; Song, C.; Cao, N.; Li, Z.; Zhou, W.; Chen, J.; Meng, L. A new mutual authentication protocol in mobile RFID for smart campus. IEEE Access 2018, 6, 60996-61005. [CrossRef]

94. Lee, C.C.; Li, C.T.; Cheng, C.L.; Lai, Y.M.; Vasilakos, A.V. A novel group ownership delegate protocol for RFID systems. Inf. Syst. Front. 2019, 21, 1153-1166. [CrossRef]

95. Munoz-Ausecha, C.; Ruiz-Rosero, J.; Ramirez-Gonzalez, G. RFID applications and security review. Computation 2019, 9, 69. [CrossRef] 